'Departamento de Enfermedades Cardiovasculares. ${ }^{2}$ Departamento de Nefrología. Escuela de Medicina Pontificia Universidad Católica de Chile.

Recibido el 26 de julio de 2013, aceptado el 17 de diciembre de

Correspondencia a: Dres. Pablo Castro / Alejandro Paredes $C$

Departamento de Enfermedades Cardiovasculares Pontificia Universidad Católica de Chile. Marcoleta \#367, $7^{\circ}$ piso, Unidad Coronaria.

Teléfono: (+56 02) 23543624 pcastro@med.puc.cl

\section{Feocromocitoma: presentación como síndrome coronario agudo. Reporte de un caso}

\author{
ANDRÉS ENRÍQUEZ ${ }^{1}$, ALEJANDRO PAREDES ${ }^{1}$, \\ RODRIGO TAGLE ${ }^{2}$, PABLO CASTRO ${ }^{1}$
}

\section{Presentation of a pheochromocytoma as an acute coronary syndrome. Report of one case}

The typical symptoms of pheochromocytoma are palpitations, sweating, headaches and hypertension. We report a 70-year-old female admitted to the hospital due to a sudden onset of precordial pain with electrocardiographic changes. After admission the patient evolved with recurrent chest pain accompanied by hypertensive paroxysms and a pheochromocytoma was suspected. Measurement of catecholamines and metanephrines confirmed the diagnosis and an abdominal magnetic resonance localized the tumor. The patient underwent surgery with successful removal of the pheochromocytoma and was discharged in good conditions.

(Rev Med Chile 2014; 142: 250-254)

Key words: Acute coronary syndrome, cardiovascular; Catecholamines; Metanephrine; Pheochormocytoma.

\section{Presentación del caso}

M ujer de 70 años que consultó en servicio de Urgencias de nuestro hospital por cuadro de dolor retroesternal opresivo, intensidad 10/10, irradiado a epigastrio, de aproximadamente 15 min de duración, asociado a sudoración profusa y palpitaciones.

Refería haber presentado episodio de similares características dos días antes, lo que motivó consulta en otro servicio de urgencias, donde fue evaluada con electrocardiograma (ECG) y enzimas cardiacas; no impresionando cuadro de origen coronario, por lo que se derivó a su domicilio. Dentro de sus antecedentes destacaba historia de hipertensión arterial de data no precisada, en tratamiento con atenolol $50 \mathrm{mg}$ al día, y cáncer de mama tratado 20 años antes con cirugía y radioterapia. La paciente no era fumadora ni tenía antecedentes familiares de cardiopatía coronaria.

$\mathrm{Al}$ ingreso, la paciente se encontraba sudorosa, pero sin dolor. La presión arterial era 100/60 mmHg y su frecuencia cardiaca $87 \mathrm{lpm}$. Al examen físico destacaban yugulares colapsables en inspiración, la auscultación cardiaca no reveló soplos ni galope y había sensibilidad a la palpación epigástrica. El resto del examen no era destacable. Al interrogatorio, la paciente refería historia de episodios similares durante los últimos 6 meses, sin clara relación con esfuerzos físicos, que en un principio eran esporádicos pero que durante la última semana se hicieron más frecuentes, casi diarios. El ECG mostró un discreto supradesnivel del segmento ST (SDST) anteroseptal con inversión de la onda T de V1 a V3, que ya era evidente en ECG tomado 2 días antes y que no fue evolutivo (Figura 1).

La radiografía de tórax mostró una silueta cardiaca de tamaño normal y no se observaban signos de congestión pulmonar. La paciente fue admitida a la unidad coronaria, con terapia de síndrome coronario agudo $\sin$ SDST. La troponina I de ingreso fue de $0,07 \mathrm{pg} / \mathrm{ml}$ (valor normal $<0,05$ ) y la CK 


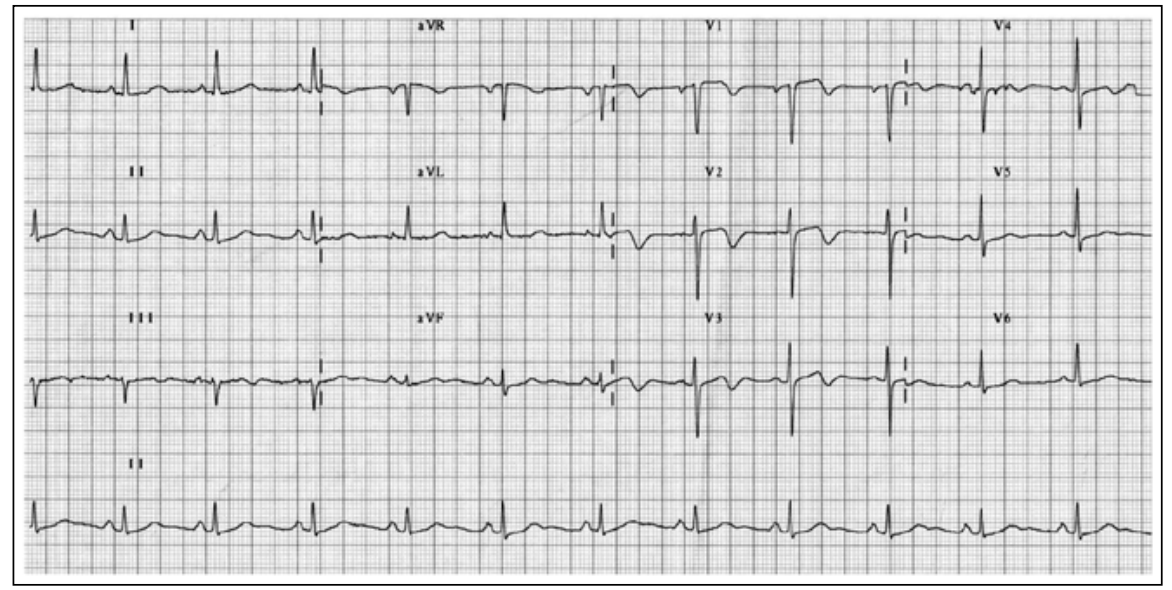

Figura 1. ECG de ingreso. Destaca mínimo supradesnivel del segmento ST anteroseptal con inversión de ondas T.

Tabla 1. Exámenes bioquímicos

\begin{tabular}{|lrl|}
\hline Catecolaminas plasmáticas & \\
Adrenalina & $9.999 \mathrm{pg} / \mathrm{ml}$ & Valor normal 20-82 pg/ml \\
Noradrenalina & $15.688 \mathrm{pg} / \mathrm{ml}$ & Valor normal 41-773 pg/ml \\
Metanefrinas urinarias & $11.328 \mathrm{ug} / 24 \mathrm{~h}$ & Valor normal $52-341 \mathrm{ug} / 24 \mathrm{~h}$ \\
$\quad$ Metanefrina & $1.548 \mathrm{ug} / 24 \mathrm{~h}$ & Valor normal 88-444 ug/24 h \\
Normetanefrina & & \\
\hline
\end{tabular}

total fue de 117 U/L con una fracción MB de 25.

A las pocas horas de ingreso la paciente evolucionó con crisis de angor intenso, 10/10, asociado a disnea, palpitaciones, cefalea, sudoración y enrojecimiento facial. Se constató alza de presión arterial hasta 260/130 mmHg y al monitor se evidenció taquicardia sinusal con extrasistolía supraventricular y ventricular frecuentes, y conducción aberrante con morfología de bloqueo completo de rama derecha de aproximadamente 5 min de duración. No hubo episodios de hipotensión ni síncope. Dado las características del episodio se sospechó el diagnóstico de feocromocitoma. Se suspendió heparina, se aportó solución fisiológica y se inició doxazosina en dosis de $1 \mathrm{mg}$ cada 12 h. Se midió durante la crisis catecolaminas plasmáticas y metanefrinas urinarias (previo al inicio de doxazosina), que fueron marcadamente elevadas (Tabla 1).

Se solicitó una resonancia magnética de abdomen (Figura 2), que confirmó el diagnóstico, evidenciando una masa suprarrenal derecha de 6 $\mathrm{cm}$ de diámetro.

El cintigrama con 123-I-metaiodobencilguanidina (MIBG) confirmó la captación en la masa descrita y no mostró metástasis (Figura 3).
Adicionalmente se realizó un ecocardiograma transtorácico, que mostró hipertrofia ventricular izquierda leve y compromiso leve de la función sistólica global, con una fracción de eyección (FE) estimada por Simpson de $42 \%$, y una coronariografía sin incidentes durante el procedimiento, que

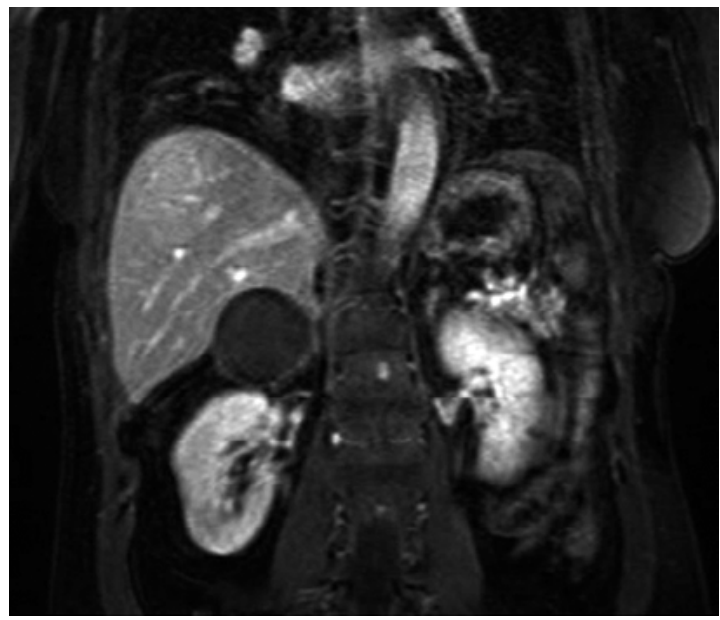

Figura 2. Resonancia magnética de abdomen mostrando masa suprarrenal derecha de $6 \mathrm{~cm}$ de diámetro mayor. 


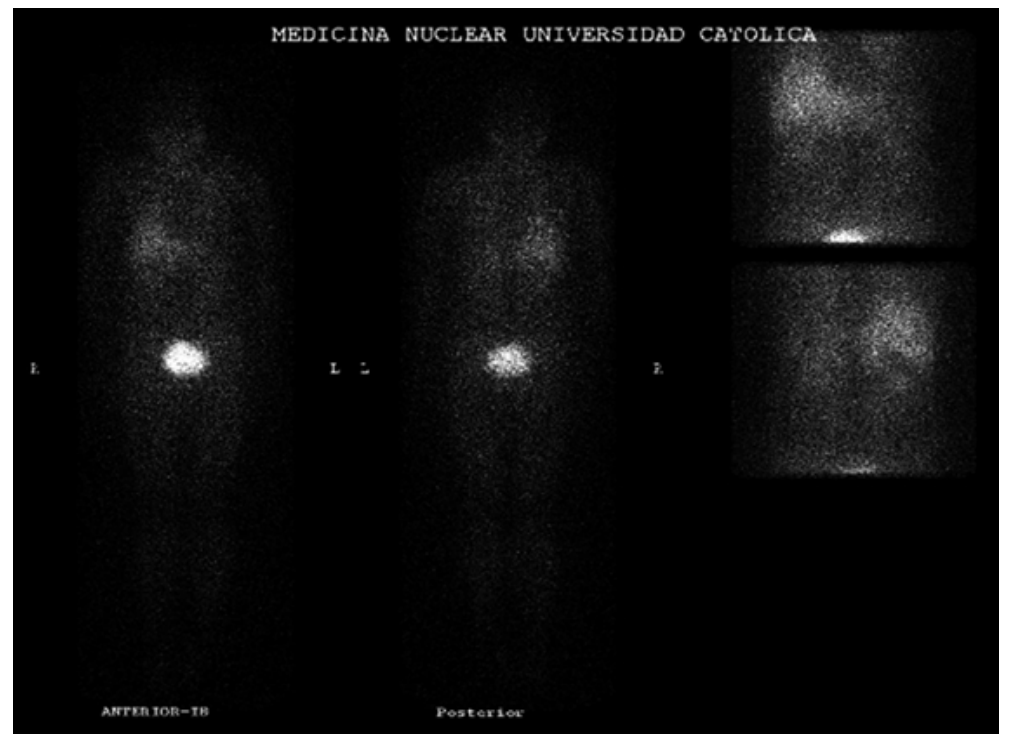

Figura 3. Cintigrama MIBG ${ }^{123}$, que no mostró evidencias de diseminación a distancia. descartó la presencia de enfermedad coronaria. La disfunción ventricular fue asumida en contexto de daño miocárdico por catecolaminas.

La paciente se preparó para la intervención quirúrgica con bloqueo $\alpha$ y $\beta$-adrenérgico escalonado con doxazosina y, luego de 2 días, propanolol. La cirugía se realizó 11 días después, sin complicaciones hemodinámicas significativas y cursó con un postoperatorio favorable. La paciente fue dada de alta a los 4 días en buenas condiciones. Desde el punto de vista clínico, cursó con buena evolución posterior con disminución progresiva en la dosis de antihipertensivos y normalización de la función ventricular en ecocardiografía de control.

\section{Discusión}

Los feocromocitomas son tumores neuroendocrinos productores de catecolaminas que se originan en las células cromafines de la médula adrenal o en los ganglios simpáticos extra-adrenales. Estos últimos son referidos como feocromocitomas extra-adrenales o paragangliomas. Es poco frecuente y se estima que su prevalencia en pacientes con hipertensión es de $0,1-0,6 \%{ }^{1}$, pero hasta $4 \%$ en pacientes con un incidentaloma adrenal ${ }^{2}$. La mayoría son esporádicos, sin embargo, cada vez más frecuentemente se detectan en síndromes heredofamiliares como: neoplasia endocrina múltiple tipo II (MEN II), enfermedad de von Hippel-Lindau, neurofibromatosis tipo I y para- gangliomas familiares asociados a mutaciones de la succinato deshidrogenasa mitocondrial (SDHB y SDHD). La evidencia ha demostrado que la regla tradicional de los 10 (10\% bilaterales, $10 \%$ extra-adrenales, $10 \%$ familiares, $10 \%$ malignos) es inexacta, ya que los extra-adrenales pueden alcanzar a $20 \%$ y la enfermedad maligna es mucho más frecuente en feocromocitomas extra-adrenales y hasta $25 \%$ son heredofamiliares ${ }^{3-5}$.

La presentación clínica es altamente variable y puede imitar los síntomas y signos producidos por muchas otras condiciones clínicas (Tabla 2), por lo que algunos lo han llamado "el gran simulador". La mayoría de las manifestaciones clínicas son consecuencia de la acción directa de las catecolaminas. La tríada sintomática clásica consiste en episodios de cefalea, sudoración y taquicardia. La mayoría de los pacientes tiene hipertensión, que es paroxística en $30 \%$ de los casos y en el resto es sostenida. Además hasta $40 \%$ de los pacientes pueden presentar hipotensión ortostática, la que se ha atribuido a la hipovolemia, al efecto vasodilatador $\beta$ de la adrenalina y al down-regulation de receptores adrenérgicos ${ }^{6}$. Los efectos metabólicos incluyen hiperglicemia, acidosis láctica y pérdida de peso.

En esta paciente, el síntoma cardinal fue el dolor torácico, que presentaba características de crisis anginosas. El diagnóstico fue sospechado al presenciar una de estas crisis, acompañadas de hipertensión y marcado correlato adrenérgico, lo que permitió el diagnóstico oportuno y la rápida 
Tabla 2. Diagnóstico diferencial de feocromocitoma

Trastornos endocrinos
- Hipertiroidismo
- Carcinoide
- Hipoglicemia
- Carcinoma medular de tiroides
- Mastocitosis
- Menopausia
Trastornos cardiovasculares
- Arritmias
- Insuficiencia cardiaca
- Cardiopatía isquémica
- Falla barorrefleja
Trastornos neurológicos
- Migraña
- Accidente cerebrovascular
- Epilepsia diencefálica
- Meningioma
- Síndrome de taquicardia postural ortostática (POTS)
Miscelános
- Porfiria
- Ansiedad o trastorno de pánico
- Efecto de medicamentos o drogas (IMAO, simpatico-
- Trastornos factícios

instauración de medidas terapéuticas.

Por otro lado, las catecolaminas pueden inducir espasmos coronario multivaso y a nivel microvascular, con disfunción coronaria microvascular aguda y transitoria por activación de $\beta$-receptores ${ }^{7}$. Estos mecanismos son responsables del atontamiento miocárdico y las anormalidades en la motilidad segmentaria ${ }^{8}$.

En cuanto a las manifestaciones cardiovasculares, además de la hipertensión arterial son frecuentes las alteraciones electrocardiográficas en el segmento ST y onda T (24\% en la serie de Liao et al) e infarto agudo de miocardio, incluso sin enfermedad coronaria9. La patogenia de esto se ha relacionado con hipoxia miocárdica funcional, espasmo coronario, cambios en la permeabilidad de la membrana para $\mathrm{Ca}^{+2} \mathrm{y} \mathrm{Mg}^{+2}$ y cambios metabólicos en la célula miocárdica, todo ello causado por metabolitos oxidativos de las catecolaminas ${ }^{10}$. Los hallazgos ecocardiográficos son variables y van desde la hipertrofia ventricular izquierda, mio- cardiopatía hipertrófica obstructiva, disfunción sistólica en sus distintos grados y shock cardiogénico, en relación a miocardiopatía dilatada. La miocardiopatía de Takotsubo es caracterizada por una disfunción sistólica transitoria de los segmentos apicales apical (síndrome de balonamiento apical) o más raramente, de los segmentos medios del ventrículo izquierdo (Takotsubo invertido), siendo el feocromocitoma más asociado a este último patrón ${ }^{7}$.

La paciente del caso se encontraba en tratamiento exclusivo con atenolol, lo que implica un riesgo inminente de complicaciones graves como disfunción miocárdica y edema pulmonar, ya que los receptores beta adrenérgicos ubicados en la vasculatura muscular no podrán ocasionar una vasodilatación en presencia de catecolaminas, y sólo los receptores alfa adrenérgicos podrán ser estimulados, produciéndose una intensa vasoconstricción periférica y un marcado aumento de la resistencia vascular sistémica ${ }^{11}$.

Ante la sospecha clínica de feocromocitoma el paso inicial para el diagnóstico es la confirmación bioquímica de la producción excesiva de catecolaminas. Los tests bioquímicos disponibles incluyen medición de catecolaminas plasmáticas y urinarias, metanefrinas (metanefrina y normetanefrina) plasmáticas y urinarias y ácido vanililmandélico (VMA) en orina. Otras pruebas menos utilizadas por su sensibilidad y especificidad incluyen cromogranina $\mathrm{A}$, neuropéptido $\mathrm{Y}$, prueba de supresión con clonidina, entre otras. $\mathrm{La}$ evidencia acumulada sugiere que las metanefrinas plasmáticas y metanefrinas urinarias fraccionadas son los tests más sensibles y específicos para el diagnóstico y, por tanto, los más adecuados para excluir con seguridad la presencia de un feocromocitoma. Los niveles de corte para considerar una prueba positiva dependen del laboratorio de referencia consultado. Además, se deben tener algunas consideraciones respecto a situaciones clínicas, fármacos, forma en la toma de muestras que pueden alterar los niveles plasmáticos o urinarios de dichas sustancias.

El segundo paso, después de la confirmación bioquímica, es localizar el tumor, lo que se realiza mediante técnicas de imagen como la tomografía axial computada (TAC) o la resonancia magnética nuclear (RMN) de abdomen. Ambos exámenes tienen alta sensibilidad (98-100\%), pero una especificidad limitada (70-80\%). El cintigrama 
con MIBG es una técnica de imágenes funcional que se basa en la capacidad del MIBG, un análogo de la norepinefrina, de ser captado por el tejido adrenal. Tiene mayor especificidad que el TAC y la RMN (95-100\%) y es útil en la identificación de tumores extra-adrenales y metástasis. Está especialmente indicado si el TAC o RMN son negativos en presencia de evidencia clínica y bioquímica de feocromocitoma. También debe considerarse en feocromocitomas adrenales $>5 \mathrm{~cm}$, debido al mayor riesgo de malignidad ${ }^{12}$.

El tratamiento definitivo del feocromocitoma es la excisión quirúrgica del tumor. Actualmente, la cirugía laparoscópica es la técnica de elección, con las ventajas sobre la cirugía abierta de una menor estadía hospitalaria, menos dolor postoperatorio y mejores resultados cosméticos ${ }^{13}$. De importancia crítica es la conducta y el abordaje terapéutico perioperatorio y anestésico de estos pacientes, que ha permitido reducir la mortalidad desde $50 \%$ a menos de $3 \%$ en la actualidad ${ }^{14}$. El objetivo principal del manejo perioperatorio es normalizar la presión arterial y la frecuencia cardiaca, corregir la depleción de volumen y prevenir las graves complicaciones cardiovasculares de las catecolaminas durante la cirugía (crisis hipertensiva, arritmias, edema agudo pulmonar e isquemia miocárdica). La mayoría de los esquemas terapéuticos incluyen bloqueo alfa-adrenérgico con doxazosina, que previene la vasoconstricción inducida por catecolaminas, seguido después de un par de días del inicio cuidadoso de betabloqueadores selectivos.

Post cirugía los pacientes requieren monitorización durante las primeras $24 \mathrm{~h}$ en una unidad de cuidados intensivos o intermedios. Las principales complicaciones son la hipotensión postoperatoria por caída brusca en los niveles de catecolaminas circulantes en presencia de bloqueo alfa y la hipoglicemia, que se debe a hiperinsulinemia de rebote al eliminar el efecto contrarregulador de las catecolaminas ${ }^{15}$. La hipotensión se trata con aporte de volumen y, eventualmente, con el uso de efedrina endovenosa, mientras que la hipoglicemia se trata con infusión de glucosa.

En conclusión, el feocromocitoma puede presentarse por crisis de dolor torácico con características anginosas y debe considerarse dentro del diagnóstico diferencial, especialmente cuando se asocia a hipertensión paroxística o se acompaña de síntomas adrenérgicos sugerentes.

\section{Referencias}

1. Goldstein DS, Eisenhofer G, Flynn JA, Wand G, Pacak K. Diagnosis and localization of pheochromocytoma. Hypertension 2004; 43 (5): 907-10.

2. Mantero F, Terzolo M, Arnaldi G, Osella G, Masini AM, Ali A, et al. A survey on adrenal incidentaloma in Italy. Study Group on Adrenal Tumors of the Italian Society of Endocrinology. J Clin Endocrinol Metab 2000; 85 (2): 637-44.

3. Petri BJ, van Eijck $\mathrm{CH}$, de Herder WW, Wagner A, de Krijger RR. Phaeochromocytomas and sympathetic paragangliomas. Br J Surg 2009; 96 (12): 1381-92.

4. Donckier JE, Michel L. Phaeochromocytoma: state-ofthe-art. Acta Chir Belg 2010; 110 (2): 140-8.

5. Neumann HPH, Bausch B, McWhinney SR, Bender BU, Gimm O, Franke G, et al. Germ-Line Mutations in Nonsyndromic Pheochromocytoma. N Engl J Med 2002; 346 (19): 1459-66.

6. Tagle R, Acosta P, Valdés G. [Orthostatic hypotension: an unusual manifestation of pheochromocytoma]. Rev Med Chile 2003; 131 (12): 1429-33.

7. Cardillo MT, Bona RD, Caroli A, Valentini AL, Biasucci LM. A case of unusual acute coronary syndrome. Am J Emerg Med 2013; 31 (4): 758 e1-2.

8. Galetta F, Franzoni F, Bernini G, Poupak F, Carpi A, Cini G, et al. Cardiovascular complications in patients with pheochromocytoma: A mini-review. Biomedicine \& Pharmacotherapy 2010; 64 (7): 505-9.

9. Wei-Ber L, Chu-Feng L, Ching-Wen C, Chia-Te K, ChiWei L. Cardiovascular manifestations of pheochromocytoma. The American journal of emergency medicine 2000; 18 (5): 622-5.

10. Behonick GS, Novak MJ, Nealley EW, Baskin SI. Toxicology update: the cardiotoxicity of the oxidative stress metabolites of catecholamines (aminochromes). J Appl Toxicol 2001; 21 Suppl 1: S15-22.

11. Sibal L, Jovanovic A, Agarwal SC, Peaston RT, James RA, Lennard TWJ, et al. Phaeochromocytomas presenting as acute crises after beta blockade therapy. Clinical Endocrinology 2006; 65 (2): 186-90.

12. Chrisoulidou A, Kaltsas G, Ilias I, Grossman AB. The diagnosis and management of malignant phaeochromocytoma and paraganglioma. Endocr Relat Cancer 2007; 14 (3): 569-85.

13. Bravo EL, Tagle R. Pheochromocytoma: State-of-the-Art and Future Prospects. Endocr Rev 2003; 24 (4): 539-53.

14. Van Braeckel P, Carlier S, Steelant PJ, Weyne L, Vanfleteren L. Perioperative management of phaeochromocytoma. Acta Anaesthesiol Belg 2009; 60 (1): 55-66.

15. Bravo EL, Tarazi RC, Gifford RW, Stewart BH. Circulating and urinary catecholamines in pheochromocytoma. Diagnostic and pathophysiologic implications. N Engl J Med 1979; 301 (13): 682-6. 